\title{
New Results from the SMA
}

\author{
James M. Moran \\ Harvard-Smithsonian Center for Astrophysics, \\ 60 Garden St., Cambridge, MA 02138 USA
}

\begin{abstract}
The Submillimeter Array (SMA) is nearing completion in "millimeter" valley just below the summit of Mauna Kea in Hawaii. Numerous astronomical observations have been undertaken in the partially transparent bands between 0.45 and $1.3 \mathrm{~mm}$. The status of the instrument is described in this paper, and examples of images that have been made with the partially completed Array of the dust and gas envelopes of a high mass protostellar object (IRAS 18089-1732), a low mass young star (LkCa15), and three evolved stars (CRL 2688, V Hydrae, and IRC+10216) are presented.
\end{abstract}

\section{Introduction}

The idea of an instrument to make high resolution images at submillimeter wavelengths was part of the initiative of Irwin Shapiro in 1983 as the newly appointed Director of the Center for Astrophysics, to work towards high resolution imaging capability across a broad range of the electromagnetic spectrum. At that time, arrays were in operation at Owens Valley Radio Observatory (OVRO), Berkeley Illinois Maryland Association (BIMA), Institut de Radioastronomie Millimétrique (IRAM), and Nobeyama Radio Observatory (NRO) in the three millimeter wavelength band. Exploratory observations at lower resolution with single aperture telescopes had been made at submillimeter wavelengths, especially with bolometric detectors. The challenge for high resolution submillimeter wavelength imaging was to place an instrument at a sufficiently dry site to allow operation in the partially transparent submillimeter bands and to develop sensitive receivers. The technical viability and expected scientific impact was studied at SAO and a report was written (Moran et al. 1984). Start up funding for a receiver development lab was received in 1989. The Major Instrumentation Program at SAO, initiated in 1992, was used to fund the design and construction of the Submillimeter Array (SMA) as well as to upgrade the MMT facility in Arizona. In 1996, the Institute of Astronomy and Astrophysics of the Academia Sinica of Taiwan (ASIAA) joined the SMA project and provided an additional two antennas to the initial complement of six.

\section{Technical Capabilities}

At this time (October 2003), the SMA is nearing completion (see Figure 1) and a number of scientific programs have already been undertaken with the partially 
completed Array. Table 1 summarizes the basic characteristics of the Array. Table 2 gives the sensitivity of the instrument as a function of frequency and resolution for typical weather conditions. The collecting area of the Array is relatively modest so that the continuum sensitivity is only about $1 \mathrm{mJy}$ in eight hours at $0.87 \mathrm{~mm}$. However, because of the frequency squared dependence of the Rayleigh-Jeans part of the blackbody spectrum the brightness temperature sensitivity is very good compared to instruments operating at lower frequencies (i.e. the brightness temperature noise level scales as $\nu^{2}$ and as $\theta_{f}^{-2}$ where $\nu$ is the frequency and $\theta_{f}$ is the angular resolution).

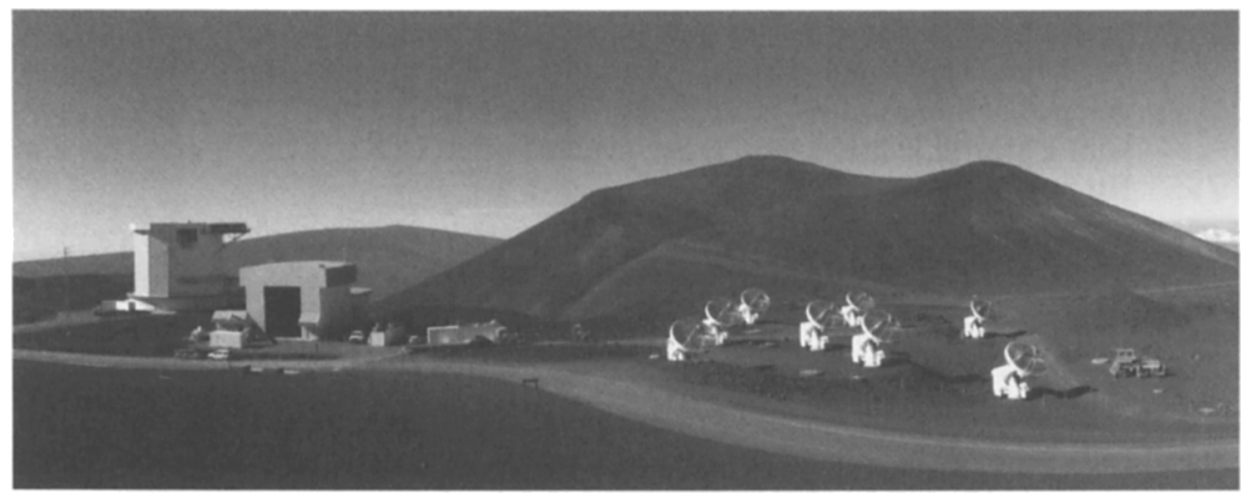

Figure 1. The SMA on October 1, 2003. The eight antennas of the Array are in a hybrid configuration of the two innermost rings. At the far right is the vehicle used to transport the antennas among the 24 pads. The antennas were assembled in the building on the left with the large door. At the far left is the J.C. Maxwell telescope which, with the Caltech Submillimeter Observatory telescope (out of view on the left), will operate as part of the Array beginning in 2005. Pu'u Poli'ahu is immediately behind the Array and Mauna Loa is in the distance behind the SMA assembly building. (Photo by J. Weintroub)

The accurate alignment of the reflector surfaces is a prime achievement of the Array. One of the antennas has been successfully set to the design specification of $12 \mu \mathrm{m}$ (See Figure 2). The SMA antennas have the most precise surfaces of any radio astronomical antennas in their size class.

An important characteristic of the instrument will be its ability to make observations at two frequencies simultaneously. This provides a convenient way to obtain polarization measurements, increased sensitivity, and correction of the phase of the higher frequency visibility measurements. Figure 3 shows the first result of two frequency operation. 
Table 1. SMA Characteristics and Status in October 2003

\begin{tabular}{|c|c|}
\hline Antennas & $\begin{array}{l}8 \times 6-\mathrm{m} \text { diameter } \\
\text { machined aluminum panels; } \\
\text { Carbon fiber backup structures; } \\
12 \mu \mathrm{m} \text { rms surface accuracy } \\
\text { (current accuracies } 12-27 \mu \mathrm{m} \text { ) }\end{array}$ \\
\hline Configuration & $\begin{array}{l}24 \text { pads in } 4 \text { rings } \\
(9 \text { pads tested })\end{array}$ \\
\hline Baselines & $\begin{array}{l}\text { 8-508 m } \\
\text { (maximum tested: } 120 \mathrm{~m} \text { ) }\end{array}$ \\
\hline Receivers & $\begin{array}{l}\text { Maximum of } 8 \text { per cryostat ( } 2 \text { bands simultaneously) } \\
\text { Bands: } \\
177-256 \mathrm{GHz} \text { ( } 8 \text { available) } \\
256-360 \mathrm{GHz}(8 \text { available }) \\
320-420 \mathrm{GHz}(2004) \\
400-520 \mathrm{GHz}(2006) \\
600-720 \mathrm{GHz}(6 \text { available }) \\
780-920 \mathrm{GHz}(2007)\end{array}$ \\
\hline Correlator & $\begin{array}{l}\text { Hybrid analog - digital design: } \\
\text { analog filtering to } 104 \mathrm{MHz} \text {, effective digital } \\
\text { sampling, with time multiplexing rate } \\
208 \mathrm{MHz}, 2 \mathrm{GHz} \text { total bandwidth. } \\
\text { Standard mode: } 2 \mathrm{GHz} \text { bandwidth, } 0.8 \mathrm{MHz} \\
\text { channel spacing, } 28 \text { baselines, two bands } \\
\text { Higher resolution available at reduced } \\
\text { bandwidth. }\end{array}$ \\
\hline Data rate & $\begin{array}{l}\text { Maximum } 10 \text { Gbyte/day } \\
\text { Current operation: } 1 \text { receiver, } 1 \mathrm{GHz} \text { bandwidth, } \\
6 \text { antennas, (i.e., } 12 \text { percent capacity) }\end{array}$ \\
\hline
\end{tabular}

\section{Scientific Results}

The problems that can be studied at submillimeter wavelengths are described in reviews by Sargent and Welch (1993), and Shaver (1996), and others. In particular, the high frequency or short wavelength end of the radio spectrum is very sensitive to thermal emission from cool dust and optically thin emission from spectral lines. Since the emissivity of dust grains varies as $\nu^{1-2}$, the flux density from dust in the Rayleigh-Jeans regime will vary as $\nu^{3-4}$. This means that the envelopes of many dust enshrouded star forming regions that are invisible at longer wavelengths and optically thick at optical wavelengths will be partially transparent in the submillimeter regime and readily detectable without obscuring completely the embedded sources. For optically thin spectral lines, the flux density increases as $\nu^{4}$ because the Einstein A coefficient for electric 
Table 2. SMA Sensitivity

\begin{tabular}{lccccc}
\hline $\begin{array}{l}\nu / \lambda^{b} \\
(\mathrm{GHz} / \mathrm{mm})\end{array}$ & $\begin{array}{r}\mathrm{T}_{\text {sys }}{ }^{c} \\
(\mathrm{~K})\end{array}$ & $\begin{array}{c}\theta_{f}{ }^{d} \\
\left(^{\prime \prime}\right)\end{array}$ & $\begin{array}{c}\theta_{r}{ }^{e} \\
\left(^{\prime \prime}\right)\end{array}$ & $\begin{array}{c}\Delta T^{f} \\
(\mathrm{~K})\end{array}$ & $\begin{array}{c}\Delta S^{g} \\
(\mathrm{mJy})\end{array}$ \\
\hline $230 / 1.3$ & 150 & 54 & 5.9 & 0.02 & 0.4 \\
& & & 0.4 & 5 & \\
$345 / 0.87$ & 340 & 36 & 3.9 & 0.04 & 1.0 \\
& & & 0.25 & 10 & \\
$460 / 0.65$ & \multirow{2}{*}{1870} & 27 & 2.9 & 0.20 & 5.7 \\
& & & 0.18 & 49 & \\
$690 / 0.43$ & 2640 & 18 & 2.0 & .024 & 8.7 \\
& & & 0.12 & 62 & \\
$805 / 0.37$ & 4870 & 15 & 1.7 & 0.44 & 17.0 \\
& & & 0.10 & 110 & \\
\hline
\end{tabular}

${ }^{a}$ Adapted from Moran (1998). Noise levels are $1 \sigma$ values, based on an integration time of 8 hours.

${ }^{b}$ Band frequency/wavelengths are denoted by the transitions of the CO line they contain.

${ }^{c}$ SSB system temperatures with $1 \mathrm{~mm}$ of precipitable water and 1.5 air masses (opacities of $0.075,0.26,1.2,1.2$, and 1.2 for $230,345,460,690$, and $805 \mathrm{GHz}$ ). The (DSB) receiver temperatures are $30,50,70,180$, and $500 \mathrm{~K}$ respectively. System temperatures include a room temperature loss of 5 percent in coupling to the antenna.

${ }^{d}$ Field of view.

${ }^{e}$ Resolution for $32 \mathrm{~m}$ (smallest) and $508 \mathrm{~m}$ (largest) configurations.

$f$ The Rayleigh-Jeans equivalent rms noise level in brightness temperature with $1 \mathrm{~km} \mathrm{~s}^{-1}$ resolution.

${ }^{g}$ Flux density rms noise level for a $2 \mathrm{GHz}$ bandwidth.

dipole transitions scales as $\nu^{3}$ and the Boltzmann factor for lines in the rotational ladder of a molecule scales as $\nu$. Hence, a plethora of molecules can be studied in the submillimeter band (see Figure 8). For these reasons, astronomical targets such as terrestrial planetary bodies, gas in molecular clouds in the Milky Way and external galaxies, and high redshift "SCUBA" galaxies can be readily studied. In addition, the flux density from very compact optically thick synchrotron sources varies as $\nu^{2-2.5}$. Many interesting objects, such as the radio source Sgr $\mathrm{A}^{*}$, are particularly prominent at submillimeter wavelengths (Zhao et al. 2002).

In keeping with the scientific focus of this conference, I have concentrated the discussion of early SMA observations on those related to stars. The SMA does not have the sensitivity to detect black body photospheric emission from any but the closest main sequence stars. Its application is primarily to stars with extended envelopes, including newly formed stars and evolved stars. Several examples of early observations are described below. 

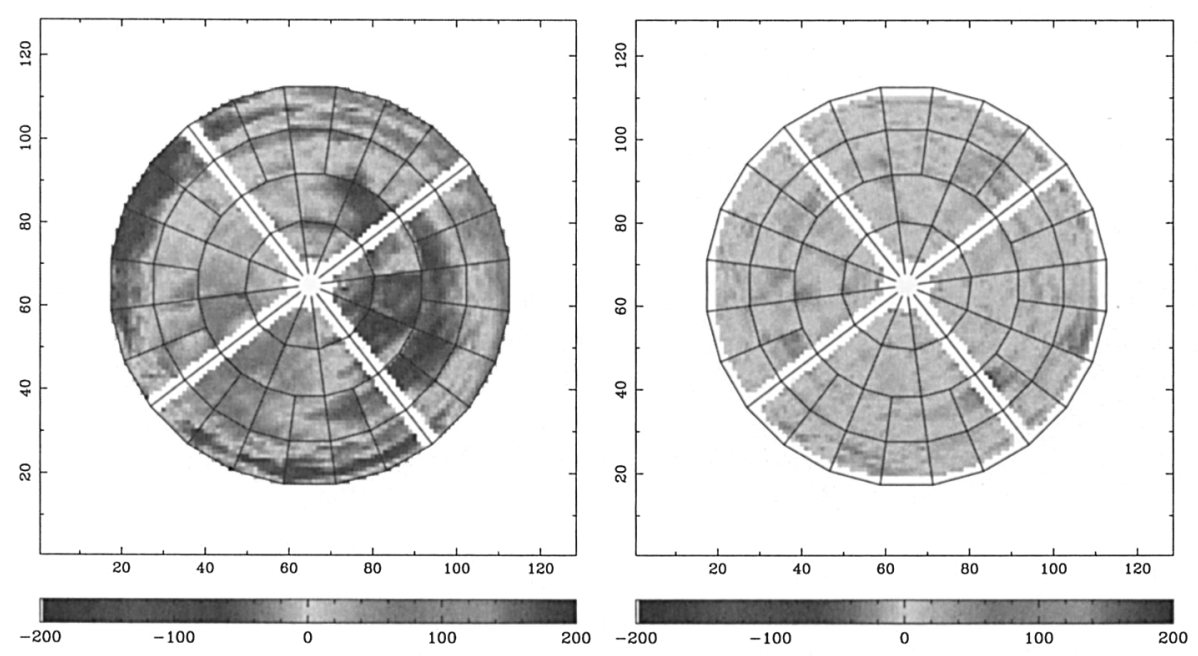

Figure 2. The reflector of each antenna has 72 machined aluminum panels with rms surface accuracies of $5 \mu \mathrm{m}$ or smaller. The figure of each of the reflectors was accurately determined by the technique of radiometric holography by use of a $230 \mathrm{GHz}$ beacon mounted on the pier of the Subaru telescope. The figure of a reflector after its initial mechanical adjustment to an accuracy of $65 \mu \mathrm{m}$, is shown on the left. Its figure after the final adjustment to $12 \mu \mathrm{m}$ accuracy is shown on the right. (From T.K. Sridharan, private communication)

\subsection{The EGG Nebula (CRL 2688)}

The Egg nebula is a protoplanetary nebula that evolved from an AGB star about $10^{2}$ years ago. The image of the CO emission in the Egg Nebula was among the first images made with the SMA when it began operation in 2001 at $345 \mathrm{GHz}$ with four antennas. The image of the $\mathrm{CO}(3-2)$ emission is shown in Figure 4, along with the optical image from the HST. The CO image shows the bipolar outflow in the direction of the optical extension and also a secondary orthogonal flow. The morphology is very similar to that reported for the $\mathrm{CO}(2-1)$ emission imaged with the IRAM Array (Cox et al. 2000).

\subsection{Hydrae}

V Hydrae is a carbon star located at a distance of $380 \mathrm{pc}$, which is on the AGB part of the HR diagram or has recently evolved into a post AGB stage. It has a mass loss rate of about $10^{-6}$ solar masses per year. Low resolution observations have shown an asymmetrical envelope with high velocity emission (e.g., Knapp, Jorissen, and Young 1997). The SMA provided the first images of the $\mathrm{CO}(3-2)$ and $\mathrm{CO}(2-1)$ emission at $345 \mathrm{GHz}$ and $230 \mathrm{GHz}$ (Hirano et al. 2003) from this 
star. Data were obtained on a total of 15 baselines, which provided a resolution of four arcseconds (1500 AU). The emission shows an extended structure in the NS direction with a velocity flow of $\pm 40 \mathrm{~km} \mathrm{~s}^{-1}$ and size of $20 \mathrm{x} 12$ arcseconds (7600 x $4600 \mathrm{AU})$, as well as bipolar jets in the EW direction with velocities of $\pm 150 \mathrm{~km} \mathrm{~s}^{-1}$. Figure 5 shows the data and a simplified model of the star's circumstellar structure.

\subsection{IRC10216}

IRC10216 is an evolved star with an extended carbon rich envelope. Its chemistry has been extensively studied with millimeter wavelength interferometers. This star was the first source imaged by the SMA in the $690 \mathrm{GHz}$ band. At the time of the observations in December 2002, the Array had only three antennas in operation at $690 \mathrm{GHz}$. Phase calibration of the Array presents a major problem in this frequency band. By fortunate circumstance, Callisto, a moon of Jupiter, was only seven degrees away and served as a bright phase calibrator source of $57 \mathrm{Jy}$. The envelope is an approximately spherically symmetric outflow within which the molecular constituency changes as a function of radius. The emission from CS(14-13), imaged as a function of velocity (Young et al. 2003) and shown in Figure 6, arises close to the stellar photosphere. As with other molecular emission, the most compact emission is at the highest and lowest velocities, which arises from the caps of the shell in front of and behind the star, while the emission at the velocity of the star arises from the tangentially outflowing gas.

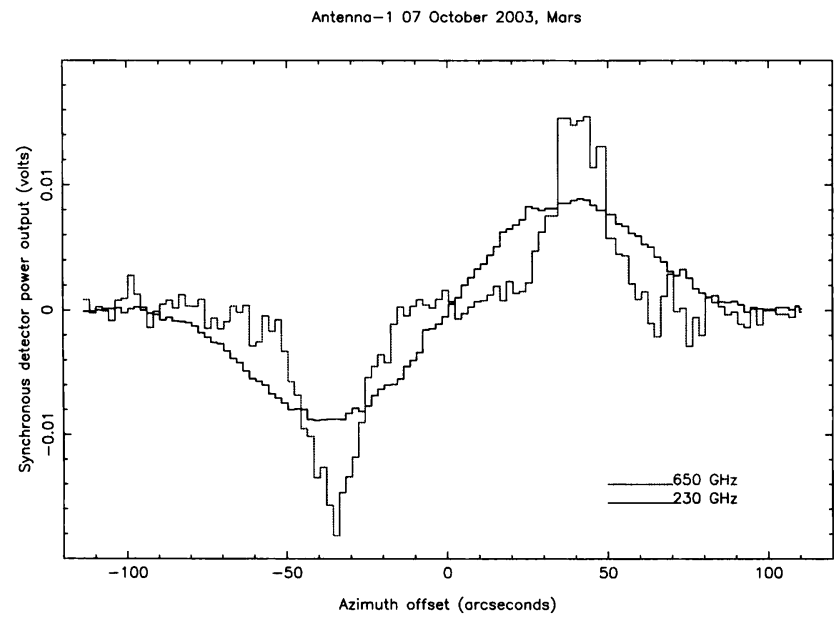

Figure 3. Scans of Mars made simultaneously at the frequencies 230 and $690 \mathrm{GHz}$, with the chopping secondary, which has a throw of 80 arcseconds, running. The optics are aligned to an accuracy of about 10 arcseconds. (From N. Patel, private communication) 


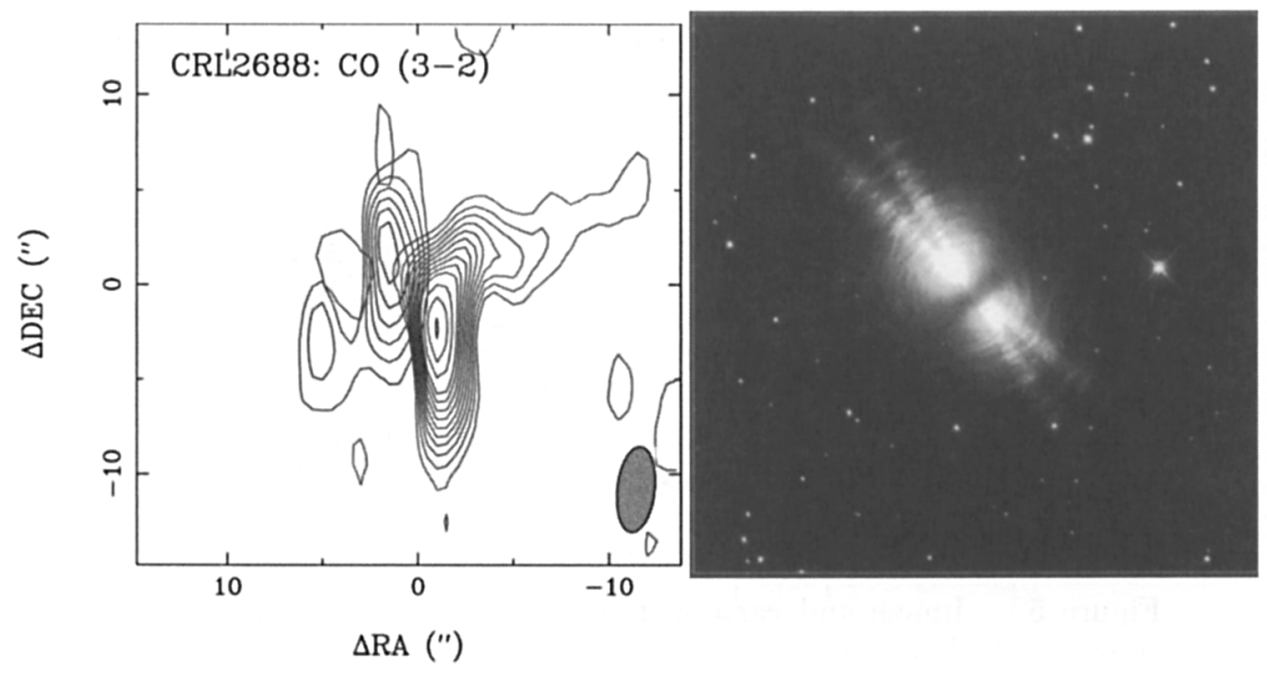

Figure 4. Right: an optical image of the Egg Nebula (CRL2688) made with the Hubble Space Telescope which shows a bipolar structure. Left: image made of the high velocity components of the $\mathrm{CO}(3-2)$ transition (dark and light contours), which shows an additional outflow along a SE-NW axis. (From N. Patel, private communication)

\subsection{LkCa 15}

LkCa 15, a K5 star located in the Taurus-Auriga molecular cloud complex at a distance of $140 \mathrm{pc}$, is one of the best studied T Tauri stars at millimeter wavelengths. Its age has been estimated to be between 3 and $12 \times 10^{6}$ years. Previous interferometric observations in the $\mathrm{CO}(2-1)$ transition by Duvert et al. (2000) and Qi et al. (2003) show a gaseous disk extending out to about $700 \mathrm{AU}$ with a velocity gradient that is a clear indication of Keplerian rotation. The disk ellipticity suggests that its inclination angle is about $60^{\circ}$. The image of the $\mathrm{CO}(3-2)$ emission made with the SMA with a resolution of about $4^{\prime \prime}$ shows the previously identified rotational signature and the distribution of the hotter gas (see Figure 7).

\subsection{IRAS 18089-1732}

IRAS $18089-1732$ is a very young high mass object, which is either a protostar or a newly formed star (Sridharan et al. 2002). Its distance is $3.6 \mathrm{kpc}$ and it has a bolometric luminosity of $10^{4.5}$ solar luminosities. Images and spectra obtained with the SMA are shown in Figure 8 (Beuther 2003).

The high spectral resolution and wide spectral bandwidth of the SMA spectrometer shows a rich molecular envelope in this object. All of the spectral lines can be imaged simultaneously and selected examples are shown in the figure. 


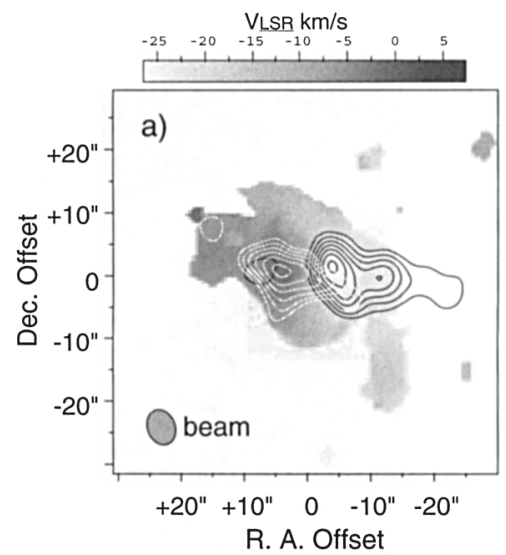

b)

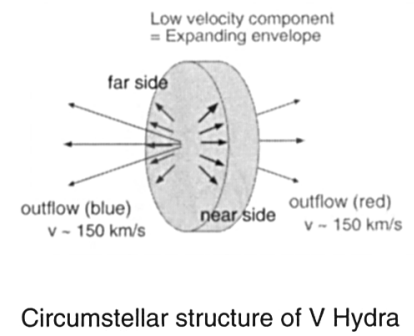

Figure 5. Image and cartoon model of V Hydrae. Left: contours show the high red-shifted (solid line) and blue-shifted (dotted line) emission in the $\mathrm{CO}(2-1)$ transition. The distribution of the mean velocity of the gas is shown in the shaded image. Right: model of the stellar envelope showing a low velocity expanding disk and a high velocity polar outflow ( $\pm 150 \mathrm{~km} \mathrm{~s}^{-1}$.) (From Hirano et al. 2003)

The HCN and SO emission, which is displaced from the core (thermal dust continuum emission) traces parts of the molecular outflow.

\section{Instrument Development and Use}

By the end of 2004, we expect that the SMA will operate smoothly with all eight antennas in the 230, 345, or $690 \mathrm{GHz}$ bands with full bandwidth of $2 \mathrm{GHz}$. There are three major projects for 2004.

The first project will be to double the data rate by allowing operation in two bands simultaneously. This goal will require doubling the IF processing capability and the correlator capacity. The equipment is in hand and the task is one of system integration. In addition to doubling the data acquisition rate, the dual frequency operation will enable a calibration strategy of using the $230 \mathrm{GHz}$ band as a phase reference for the higher band where calibration sources are weaker and the phase fluctuations caused by the atmosphere are larger. The second major project will be to build receivers for the $320-420 \mathrm{GHz}$ band (see Table 2). The $40 \mathrm{GHz}$ overlap with the $256-360 \mathrm{GHz}$ will be particularly useful. It will improve the sensitivity in a fixed integration time for observations of sources in the overlap band. Since the receivers will have orthogonal linear polarizations, complete and efficient determination of the polarization parameters of sources will be possible without the use of time-multiplexed quarterwave plates. The third project will be the integration of the JCMT and CSO telescopes into the array for observations in the $345 \mathrm{GHz}$ band. The correlator has the capacity to 

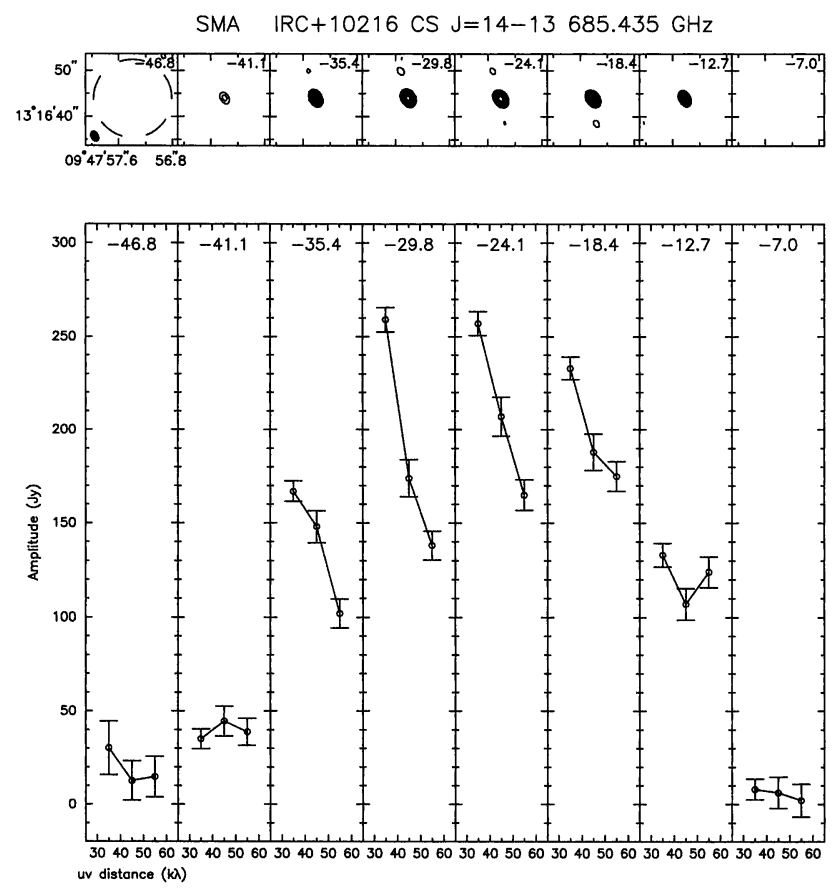

Figure 6. Top: images of IRC +10216 in the CS(14-13) transition at $685 \mathrm{GHz}$ as a function of velocity from -46.8 to $-7.0 \mathrm{~km} \mathrm{~s}^{-1}$. The leftmost panel shows the field of view imaged and the resolution beam. Bottom: fringe visibility amplitude as a function of baseline length for various velocities. Rapidly declining visibility functions indicate large component sizes. (From Young et al. 2003) 

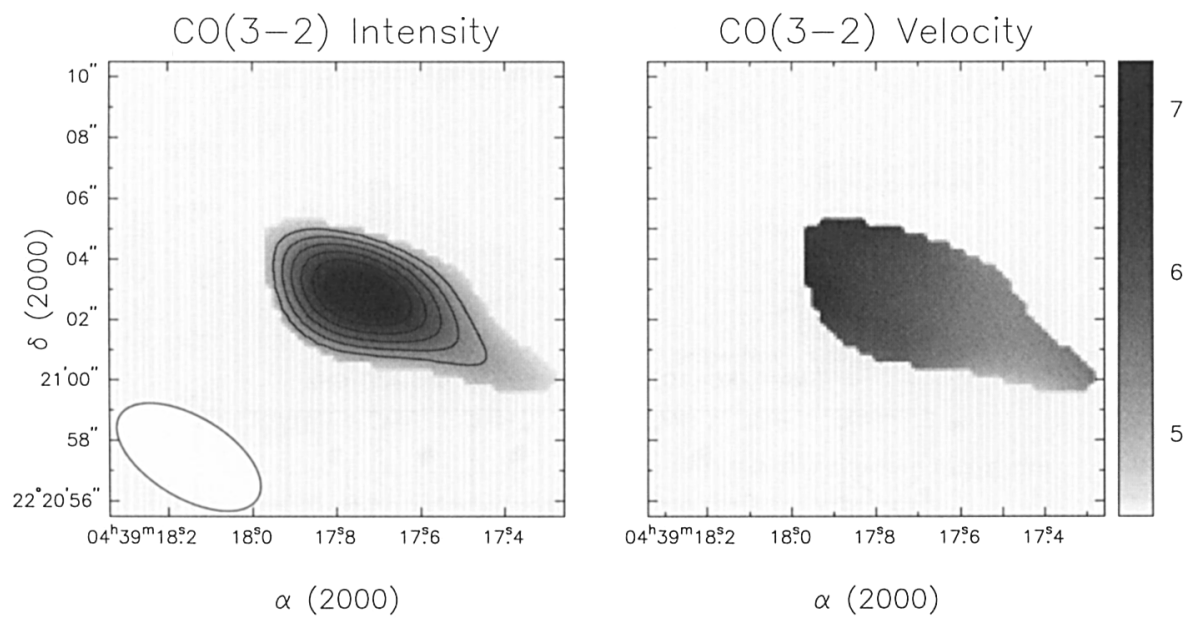

Figure 7. LkCa15. Left: Image in the $\mathrm{CO}(3-2)$ transition, with the beam shown in lower left. Right: average velocity distribution which shows a gradient indicative of rotation. (From C. Qi, private communication)

process data for 10 antennas on 45 baselines. The combined array will improve the $\mathrm{u}-\mathrm{v}$ plane coverage of the Array, increase the resolution by 50 percent, and it will more than double its collecting area. An agreement has been reached among the observatories to dedicate specific periods of 2005 for 10 station operation on projects selected by a special telescope Time Allocation Committee.

Starting in 2005, receivers for the bands $400-520 \mathrm{GHz}$ and $780-920 \mathrm{GHz}$ will be installed. In the higher band, the technology for providing sensitive SIS receivers and reliable local oscillator power sources is rapidly improving.

There is room on Mauna Kea to conveniently extend the baselines with outlying stations, which would allow for the doubling of the resolution. Such a plan is contained part of the Mauna Kea Science Reserve Master Plan (University of Hawaii, 2000). However, any such expansion would require a complete review by relevant oversight authorities.

More information about the SMA can be found on the project website (http://sma-www.cfa.harvard.edu). A restricted website, which provides daily updates of the Array status and observational results, is also maintained for interested parties (contact rcarey@cfa.harvard.edu for access).

Acknowledgments. The work described here was made possible by a large group of scientists and engineers at SAO and ASIAA. I thank H. Beuther, N. Hirano, N. Patel, C. Qi, T.K. Sridharan, D. Wilner, K. Young, and Q. Zhang for discussing their results and providing the images in this paper. 

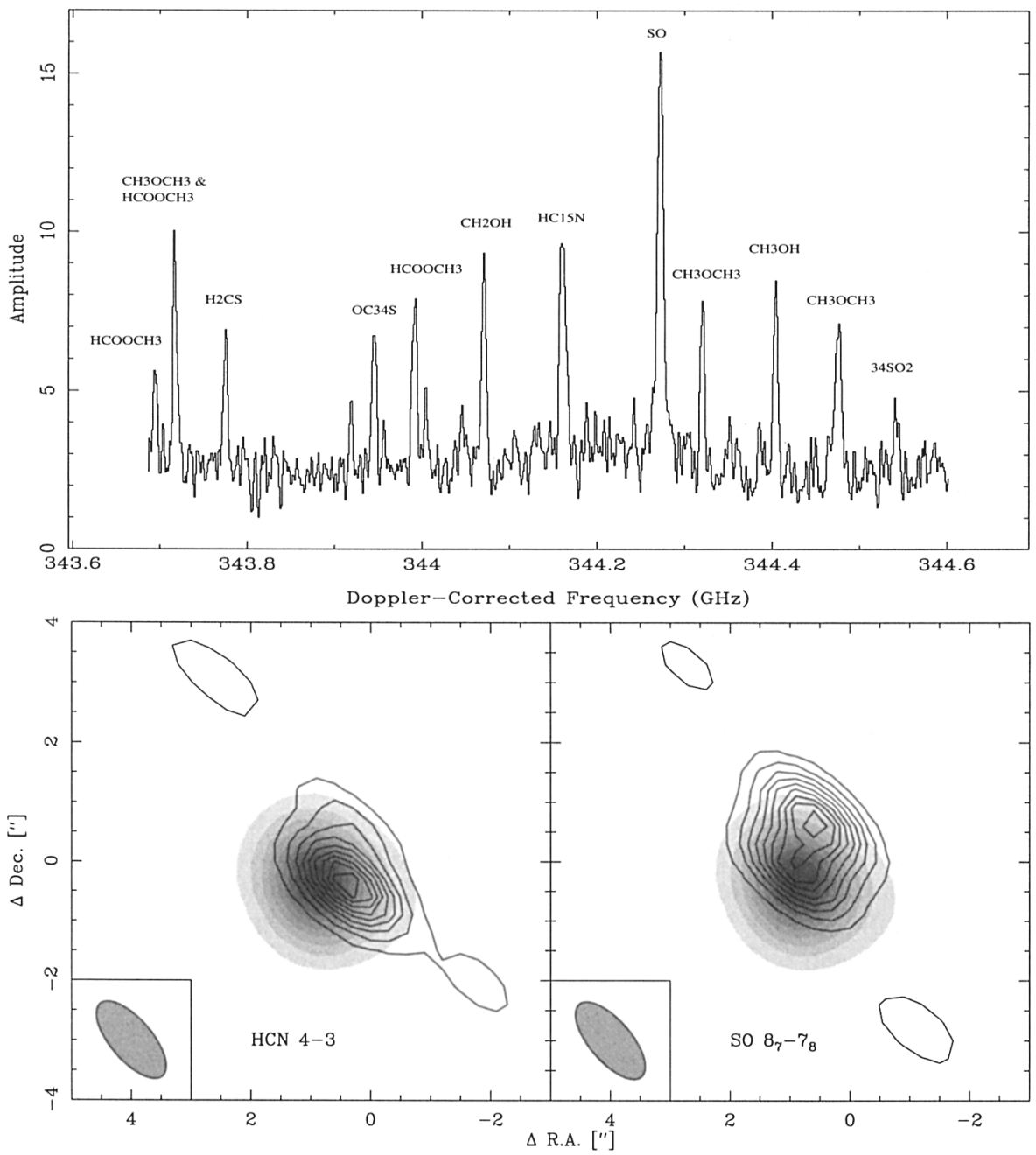

Figure 8. IRAS 18089-1732. Top: spectrum in a $1 \mathrm{GHz}$ spectrometer window around $344 \mathrm{GHz}$, which shows many molecular transitions. Lower left: intensity contours of the $\mathrm{HCN}(4-3)$ emission superimposed on the continuum emission (dust continuum) shown in gray scale. Lower right: intensity contours of $\mathrm{SO}\left(8_{8}-7_{7}\right)$ emission. (From Beuther 2003) 


\section{References}

Beuther, H. 2003, ASP 221, in Star Formation at High Angular Resolution, ed. R. Jayawardhana, M.G., Burton, \& T.L. Bourke (San Francisco: ASP)

Cox, P., Lucas, R., Huggins, P.J., Forveille, Bachiller, R., Guilloteau, S., Maillard, J.P., \& Omont, A. 2000, A\&A, 353, L25

Duvert G., Guilloteau, S., Menard, F., \& Dutrey, A. 2000, A\&A 355, 165

Hirano, N. et al. 2003, in Asymmetric Planetary Nebulae III, ASP Conference Series, ed. M. Meixner, J. Kastner, B. Balick, N. Soker, (San Francisco: ASP) in press

Knapp, G.R., Jorissen, A., \& Young, K. 1997, A\&A 326, 318

Moran, J.M. 1998, Proc. SPIE, 3357, 208

Moran, J.M., Elvis, M.S., Fazio, G.G., Ho, P.T.P., Myers, P.C., Reid, M.J., \& Willner, S.P. 1984, SAO internal report

Qi, C., Kessler, J.E., Koerner, D.W., Sargent, A.I., \& Blake, G.A. 2003 ApJ, 597,986

Sargent, A.I. \& Welch, W.J. 1993, ARAA, 31, 297

Shaver, P.A. 1996, Science with Large Millimeter Arrays, (Springer: Berlin)

Sridharan, T.K., Beuther, H., Schilke, P., Menten, K.M., \& Wyrowski, F. 2002, ApJ, 566, 931

University of Hawaii, Mauna Kea Science Reserve Master Plan 2000

Young, K.H. et al. 2003, in preparation

Zhao, J.-H., Young, K.H., Herrnstein, J.R., Ho, P.T.P., Tsutsumi, T., Lo, K.Y., Goss, W.M., \& Bower, G.C. 2003, ApJ, 586, L29 